\title{
Capacity of Ad Hoc Wireless Networks With Infrastructure Support
}

\author{
Alexander Zemlianov, Student Member, IEEE, and Gustavo de Veciana, Senior Member, IEEE
}

\begin{abstract}
We determine the asymptotic scaling for the per user throughput in a large hybrid ad hoc network, i.e., a network with both ad hoc nodes, which communicate with each other via shared wireless links of capacity $W$ bits/s, and infrastructure nodes which in addition are interconnected with each other via high capacity links. Specifically, we consider a network model where ad hoc nodes are randomly spatially distributed and choose to communicate with a random destination. We identify three scaling regimes, depending on the growth of the number of infrastructure nodes, $m$ relative to the number of ad hoc nodes $n$, and show the asymptotic scaling for the per user throughput as $\boldsymbol{n}$ becomes large. We show that when $m \lesssim \sqrt{n / \log n}$ the per user throughput is of order $W / \sqrt{n \log n}$ and could be realized by allowing only ad hoc communications, i.e., not deploying the infrastructure nodes at all. Whenever $\sqrt{n / \log n} \lesssim m \lesssim n / \log n$, the order for the per user throughput is $W m / n$ and, thus, the total additional bandwidth provided by $m$ infrastructure nodes is effectively shared among ad hoc nodes. Finally, whenever $m \gtrsim n / \log n$, the order of the per user throughput is only $W / \log n$, suggesting that further investments in infrastructure nodes will not lead to improvement in throughput. The results are shown through an upper bound which is independent of the routing strategy, and by constructing scenarios showing that the upper bound is asymptotically tight.
\end{abstract}

Index Terms-Ad hoc wireless networks, capacity scaling, hybrid wireless networks, throughput.

\section{INTRODUCTION}

I $\mathrm{N}$ THIS paper, we investigate the per user throughput that can be achieved in a hybrid ad hoc wireless network. The network consists of ad hoc nodes, that can relay information among each other via wireless links, and of infrastructure nodes, that can communicate with ad hoc nodes in a wireless manner but are also interconnected via independent high capacity wired or wireless links. We shall refer to the latter as infrastructure nodes or base stations interchangeably. There has been extensive interest in studying purely ad hoc networks, for applications involving networking of military, emergency services and, more recently, to enable the inexpensive deployment of large numbers of networked sensors in the field [1]-[4]. Since wireless units are typically energy constrained and ad hoc networks may have limited communication capacity, the addition of infrastructure or base station nodes is a natural approach to reducing

Manuscript received October 6, 2003; revised October 8, 2004. This work was supported in part by the National Science Foundation under Grant ECS0225448 .

The authors are with the Department of Electrical and Computer Engineering, University of Texas, Austin, TX 78712 USA (e-mail: zemliano@ece.utexas.edu; gustavo@ece.utexas.edu).

Digital Object Identifier 10.1109/JSAC.2004.842536 the energy and traffic burden on ad hoc nodes, while possibly increasing the system throughput. For example, one can envisage a hybrid ad hoc wireless network as a means to enable sharing of information between possibly mobile sensor nodes or gathering of sensed information toward query points on a wireline network. In this case, infrastructure nodes are leveraged by ad hoc nodes to reduce their energy burden and increase capacity. Alternatively, one can view hybrid ad hoc network model as a means to extend the communication coverage of wireless cellular infrastructure. In this case, base stations would leverage spatially distributed ad hoc nodes that are willing to relay information to increase coverage and, possibly, capacity. Note that by contrast with cellular systems in a hybrid ad hoc network not all traffic needs to be mediated through a base station, i.e., wireless nodes that wish to communicate with each other might do so directly. Thus, there are two "types" of traffic in such networks: that which is eventually mediated through the infrastructure nodes and that which is relayed in a purely ad hoc manner. In this paper, we will study the per user throughput scaling laws as the numbers of ad hoc and infrastructure nodes in a hybrid ad hoc network grow.

Related work: Let us briefly consider what is known for purely ad hoc networks [5]-[14]. The first key result is that under a reasonable interference model the aggregate transport capacity of an arbitrary network scales as $\Theta(W \sqrt{A n})$ bits-m/s in the number of nodes $n$ each placed in a region with area $A$, where each has capacity $W$ bits/s [6]. An arbitrary network is one in which the placement of ad hoc nodes and traffic loads can be selected so as to maximize the capacity. The insight offered in [6] is that system throughput is maximized when one minimizes the transmission power of each node resulting in a quadratic reduction in the interference region of a transmission. ${ }^{1}$ Minimizing the interference region permits one to schedule as many noninterfering concurrent transmissions as possible resulting in maximized transport capacity. The information theoretic results in [9] suggest that this same basic characteristic capacity scaling will continue to apply under more general communication models with more powerful interference cancellation techniques. In addition to the arbitrary network model, Gupta and Kumar [6] studies a random network model where nodes are placed at random within a given region, and choose to communicate with random destinations. In this context, they study the asymptotic scaling for the minimum per user throughput, and show it to be $\Theta(W / \sqrt{n \log n})$ bits/s. This result provides a better sense of the performance seen by individual nodes under a random traffic pattern exhibiting

\footnotetext{
${ }^{1}$ Loosely, the interference region of a transmission is the area around it, wherein it would interfere with other nodes' receptions.
} 
no spatial locality. Several follow-up papers have shown the effect of traffic locality [7], the impact of directional antennas on capacity [12], and how exploiting mobility might increase the per user throughput with varying penalties in delay [10], [13]-[15].

More recently, Liu et al. [11] have studied the aggregate throughput capacity for a hybrid network model supporting uniformly distributed traffic loads. The model assumes that the base stations are placed on a regular grid, but the ad hoc nodes are randomly distributed within the area of the network. Since the goal of [11] is to bound the aggregate throughput, in their analysis, certain users' traffic is shut off, to favor others, e.g., those with "local" traffic. Several additional interesting questions follow from this work. First, their analysis is based on partitioning bandwidth statically and in a spatially homogenous manner for purposes of supporting ad hoc and infrastructure communications. Thus, one might ask whether a spatially dependent partitioning might not result in a higher aggregate or per user throughput. Indeed, since infrastructure nodes are wired together, they will be very desirable as a shortcut for traffic that needs to traverse a long distance. Thus, one would expect infrastructure nodes to become traffic hotspots. One might then question whether allowing a larger portion of bandwidth for communication with infrastructure nodes, in regions close to such nodes, while increasing the bandwidth allocated for ad hoc communications far away from these nodes would not improve the system capacity. In [11], the capacities of two natural routing strategies between ad hoc and infrastructure nodes are studied. Thus, a second question arises as to whether a better routing strategy, e.g., one that avoids hot spots, for traffic to be carried in the ad hoc mode, might not be able to achieve a better asymptotic capacity scaling.

In contrast to [11], the work of [16] considers a hybrid network model where the positions of both base stations and ad hoc nodes are randomly selected. The authors determine the asymptotic scaling of capacity for such a network when the number of base stations scales linearly with the number of ad hoc nodes. No other scaling regimes are considered.

Contributions and organization: In this paper, we resolve some of the issues that are not treated in [11] and [16] by proving an upper bound scaling for the per user throughput in a random hybrid ad hoc network that is independent of the routing strategy. This bound is shown for networks in which infrastructure nodes are placed in any deterministic manner, including the placement on the regular grid described in [11], but ad hoc nodes are placed at random within a given region. In contrast to [11] where the range of all nodes is the same for all nodes, we allow each infrastructure node to adjust the range for each transmission. Therefore, the model is quite general in that it allows one to operate infrastructure nodes in an optimal manner. In contrast to [16], we identify three scaling regimes for the growth of the number of infrastructure nodes, $m$ with the number of ad hoc nodes $n$. In each of these regimes, we demonstrate that the upper bound is tight by specifying a particular placement for infrastructure nodes accompanied with a scheduling and routing strategy.

Specifically, we show that whenever $m \lesssim \sqrt{n / \log n}$, the per user throughput is of order $W / \sqrt{n \log n}$. This is the same as that shown for pure ad hoc networks by [6], whence, in this regime infrastructure, nodes do not enhance the capacity scaling for the hybrid network. Whenever $\sqrt{n / \log n} \lesssim m \lesssim n / \log n$ the order of the per user throughput is $W m / n$. Thus, in this case, the additional aggregate bandwidth $W m$ brought in by infrastructure nodes appears to be shared by ad hoc nodes. In fact, we demonstrate that in this regime, this scaling is achieved by letting ad hoc nodes communicate directly via infrastructure nodes, i.e., as would be the case in a cellular system. Thus, more advanced routing and scheduling schemes, e.g., proposed in [11], might achieve better leading factors, but would not change the basic asymptotic capacity scaling for such systems. Finally, whenever $m \gtrsim n / \log n$ the achievable per user throughput is of order $W / \log n$. This implies that further investments in the infrastructure do not lead to improvement in the scaling of per user throughput. In some sense, this is a result of our random uniform traffic model we are using rather than an intrinsic property of the network itself. However, it is interesting to note that due to the limited local communication capacity of ad hoc and hybrid networks fluctuations in traffic will lead to significant performance penalties. By contrast, a wireline network with shared but large links can often absorb such fluctuations through statistical multiplexing.

The organization of the paper is as follows. In Section II, we describe our network and traffic models, and define the feasible throughput for a random hybrid network. In Section III, we show an upper bound on the scaling of per user throughput which is independent of routing. Then, in Section IV, we demonstrate that upper bounds are in fact tight. Section V contains some concluding remarks.

\section{Hybrid Ad Hoc Networks: Model AND NotATION}

Model for a random hybrid network: We consider a network with $n$ ad hoc nodes, that are randomly, i.e., uniformly, placed at locations $X_{1}, X_{2}, \ldots, X_{n}$ within a disc of unit area $D$. These nodes are capable of transmitting and receiving $W$ bits/s via a wireless channel. In addition, there are $m(n)$ arbitrarily placed ${ }^{2}$ infrastructure nodes (or base stations), which are interconnected through wired or alternative wireless links which can support as much traffic as necessary. For simplicity, we shall assume base stations have a capacity of $W$ bits/s to communicate with ad hoc nodes. In contrast to ad hoc nodes, the base stations do not generate any traffic themselves, i.e., they serve purely as infrastructure which relays traffic on behalf of nodes in the ad hoc network. We will assume that the channel capacity can be split into an arbitrary number $l$ of orthogonal (noninterfering) subchannels each with $W_{j}$ bits/s such that $\sum_{j=1}^{l} W_{j}=W$. In our model, each ad hoc or infrastructure node can then send simultaneously using any number of the subchannels, to any number of receivers. However, we assume, that an ad hoc node can receive from only a single sender and cannot send and receive in the same instant. We do not impose the latter constraints on the infrastructure nodes, i.e., they are free to send and receive concurrently on orthogonal channels. In the sequel, we will consider a sequence of such networks where $n \rightarrow \infty$ and $m(n)$ grows

${ }^{2}$ For example, placed in any prespecified, but deterministic way which does not depend on the realization of ad hoc nodes. 
according to various scalings-in most cases, we write $m(n)$ simply as $m$. We will limit the cases of interest to that where $m(n) \leq n$ i.e., there are fewer infrastructure nodes than ad hoc nodes.

Uniform traffic model: We assume that each ad hoc node, say the $i$ th one at location $X_{i}$ selects a destination for its traffic as follows. It will choose a random location on the disk $S_{i}$ and will then choose the node among $X_{1}, \ldots, X_{n}$ which is closest to $S_{i}$. We shall denote the location of the $i$ th node's receiver by $Y_{i}$, and let $L_{i}$ denote the length of the segment between $X_{i}$ and $Y_{i}$. For this traffic model, it can be shown that $L_{i}, i=1, \ldots, n$ will be independent and identically distributed [6]. This traffic model exhibits no locality.

Interference model: We will adopt the protocol model for interference among nodes sharing a wireless channel, see second version in [17]. In particular, consider an ad hoc or infrastructure node located at $X_{i}$ transmitting toward another (ad hoc or infrastructure) node located at $X_{j}$ along the subchannel $s$ and using the range $r_{i}$ for this transmission. The transmission will be successful at $X_{j}$ if

$$
\left|X_{i}-X_{j}\right| \leq r_{i}, \quad \text { and } \quad\left|X_{k}-X_{j}\right| \geq(1+\Delta) r_{k}
$$

where $X_{k}$ is the location of any other node which is concurrently transmitting over the subchannel $s$, and $\Delta>0$ is some parameter. Note, that the first condition in (1) requires that the receiver is within the range of the sender, and the second-ensures that the interference caused by any other concurrent transmission in the system is limited at the receiver $X_{j}$. We will further restrict the protocol model to the case where ad hoc nodes employ a common range $r$ but allow infrastructure nodes that might not be power constrained to adjust their transmission range arbitrarily.

Per user throughput capacity: Following [11], we will extend the definition of [6] for the feasible per user throughput to the case of a random hybrid network with arbitrarily placed infrastructure nodes.

Definition 1: A throughput of $\lambda(n, m)$ per node/user is feasible if there is a placement rule for the base stations, and a spatial and temporal scheme for scheduling transmissions allowing buffering at intermediate nodes (if necessary), such that each node can send $\lambda(n, m)$ bits/s on average to its chosen destination. That is, there is $T<\infty$, such that in every interval $[(i-1) T, i T]$ every node can send $\lambda(n, m) T$ bits to its corresponding destination node.

Now, as in [6], we can define the asymptotic scaling for the per user throughput as follows.

Definition 2: The per user throughput $\Lambda(n, m)$ of a random hybrid network is of order $^{3} \Theta(\lambda(n, m))$ bits/s if there exist deterministic constants $c>0$ and $c^{\prime}<\infty$ such that

$$
\begin{gathered}
\lim _{n \rightarrow \infty} \mathbb{P}(\Lambda(n, m)=c \lambda(n, m) \text { is feasible })=1 \\
\liminf _{n \rightarrow \infty} \mathbb{P}\left(\Lambda(n, m)=c^{\prime} \lambda(n, m) \text { is feasible }\right)<1 .
\end{gathered}
$$

These two conditions can be interpreted as asymptotic lower and upper bounds over random realizations for the locations of ad hoc nodes and destinations of the traffic.

\footnotetext{
${ }^{3}$ We will use the asymptotic notation as discussed in [18], i.e., $O(g(n))$, $\Omega(g(n)), \Theta(g(n))$, and $o(g(n))$ and $\omega(g(n))$.
}

\section{UPPER BOUND ON PER USER THROUGHPUT}

Our upper bound on per user throughput for random hybrid networks will draw on various results in previous work. So, we shall start by providing a summary of several key results we will use in this paper.

\section{A. Background Results}

It can be shown that the Protocol model for interference (1) requires that whenever two simultaneous transmissions occur on the same subchannel and are successfully recovered, the discs of certain radii centered in the receivers must be disjoint [6]. Thus, each successful transmission will necessarily occupy a portion of a total area of the disc placing a constraint on the number of successful receptions that can occur on a given subchannel. The first result below is an adaptation of [6, Lemma 5.4], and provides a bound on the number of simultaneous successful transmissions that can occur in a pure ad hoc network.

Lemma 1: Consider a network with $n$ ad hoc nodes arbitrarily placed within the disc of unit area and let all the nodes use the a common transmission range $r$ for their transmissions. Under the Protocol Model (1) the number of successful simultaneous transmissions $n_{\text {sim }}$ that can occur on a given subchannel is upper bounded by

$$
n_{\text {sim }} \leq \frac{c_{1}}{\Delta^{2} r^{2}}
$$

where $c_{1}>0$ is some constant, independent of $\Delta$ and $n$.

Lemma 1 also shows that the number of simultaneous transmissions in a pure ad hoc network will be maximized, whenever the common transmission range is made as small as possible. There is, however, a limit on how small this range can be while still keeping network connectivity. This limit is identified by the following theorem, proven in [19].

Theorem 1: Let $n$ nodes be randomly placed on the disc of unit area according to a uniform distribution. Assume that two nodes are connected if the distance between them is smaller than the "connectivity range" $\rho(n)$, where

$$
\pi \rho^{2}(n) \triangleq \frac{\log n+c(n)}{n} .
$$

Then, all nodes are connected in a single cluster with probability 1 as $n \rightarrow \infty$ if and only if $c(n) \rightarrow \infty$.

The next result, which we prove in Appendix I, establishes a lower bound on the minimum range which would allow connectivity in a random hybrid network where the infrastructure nodes are placed arbitrarily.

Proposition 1: Let $n$ nodes be randomly placed on the disc of unit area according to a uniform distribution and let $m$ additional nodes be placed arbitrarily. Let $r(n, m)$ be a "connectivity range," i.e. the distance within which two nodes are assumed to be connected. Assume that $r(n, m)$ is chosen so that all $n+m$ nodes form a single connected cluster with probability 1 as $n \rightarrow \infty$. Then, $r(n, m)$ satisfies

$$
r(n, m)=\left\{\begin{array}{ll}
\Omega\left(\sqrt{\frac{\log n}{n}}\right), & \text { if } m=o\left(\frac{n}{\log n}\right) \\
\Omega\left(\frac{1}{\sqrt{m}}\right), & \text { if } m=\Omega\left(\frac{n}{\log n}\right)
\end{array} .\right.
$$


Note that Proposition 1 establishes a lower bound on the required connectivity range under different joint scalings for the number of ad hoc and infrastructure nodes.

\section{B. Derivation of the Upper Bound}

We will define three scaling regimes for the number of infrastructure and ad hoc nodes
i) $m(n)=O\left(\sqrt{\frac{n}{\log n}}\right)$
ii) $m(n)=\omega\left(\sqrt{\frac{n}{\log n}}\right)$ and $m=O\left(\frac{n}{\log n}\right)$
iii) $m(n)=\omega\left(\frac{n}{\log n}\right)$.

Note that Regime i) corresponds to the case where $m \lesssim \sqrt{n / \log n}$, Regime ii)—to the case where $\sqrt{n / \log n} \ll$ $m \lesssim(n / \log n)$, and Regime iii)—to the case where $m \gg(n / \log n)$. Our upper bounds on the asymptotic scaling for the per user throughput in these three regimes are formally stated in the following theorem.

Theorem 2: The throughput per user $\Lambda(n, m)$ in a random hybrid network under the protocol model is such that for some $c^{\prime}>0$ independent of $n$ and $m$, we have

$$
\lim _{n \rightarrow \infty} \mathbb{P}\left(\Lambda(n, m)=c^{\prime} \lambda(n, m) \text { is feasible }\right)=0
$$

where

$$
\lambda(n, m)= \begin{cases}\frac{W}{\sqrt{n \log n}} & \text { in Regime i) } \\ \frac{W m}{n} & \text { in Regime ii) } \\ \frac{W}{\log n} & \text { in Regime iii) }\end{cases}
$$

We prove the theorem via two propositions as follows.

Proposition 2: The throughput per user, $\Lambda(n, m)$ for a random hybrid network under the Protocol model satisfies

$$
\Lambda(n, m)=\left\{\begin{array}{ll}
O\left(\frac{W m}{n}\right), & \text { if } m=\Omega\left(\sqrt{\frac{n}{\log n}}\right) \\
O\left(\frac{W}{\sqrt{n \log n}}\right), & \text { if } m=O\left(\sqrt{\frac{n}{\log n}}\right)
\end{array} .\right.
$$

Proof: For simplicity, we assume transmissions are slotted, with slots of length $\tau$ s. By definition a throughput $\lambda$ is feasible if over a large period of time, say $[0, T]$, each node would be able to send $\lambda T$ bits over $T / \tau$ transmission slots. Note that each node may send some (or all) of its $\lambda T$ bits using base station infrastructure and must send the remainder in ad hoc mode via multihop relaying. The idea of our proof is to bound the ad hoc traffic burden on the network that $n$ nodes would produce.

We will allow nodes to send over $l$ noninterfering subchannels each with bandwidth $W_{j}$ such that $\sum_{j=1}^{l} W_{j}=W$. Since a hybrid network consists of both nodes and base stations with the same capability sharing these subchannels, the maximum bits per second on the uplink to a particular base station is at most $W$ bits/s. Since there are $m$ such base stations, the total uplink flow in a given slot is at most $W m \tau$ bits.
Suppose that $\kappa_{i}^{u}(t) W m \tau$ bits transmitted by node $i$ are relayed on uplinks to base stations during time slot $t$. Let $\bar{\kappa}_{i}^{u}$ denote the time average of the fractional uplink base station burden $\kappa_{i}^{u}(t)$ associated with node $i$, i.e.,

$$
\bar{\kappa}_{i}^{u} \equiv \frac{1}{T} \sum_{t=1}^{\left\lceil\frac{T}{\tau}\right\rceil} \kappa_{i}^{u}(t) \tau .
$$

This quantity must satisfy the following constraints:

$$
\sum_{i=1}^{n} \bar{\kappa}_{i}^{u} \leq \frac{1}{2} \quad \text { and } \quad \bar{\kappa}_{i}^{u} W m \leq \lambda .
$$

The first constraint is due to flow conservation, i.e., the aggregate uplink burden on base stations can not exceed $1 / 2$ since these same bits will need to be sent by base stations on downlinks. The second inequality must hold true for all nodes $i$ since each node's feasible throughput will at least exceed its time average uplink burden on the base stations.

The total number of bits $N_{i}$ transmitted in "ad hoc" manner from node $i$ to its destination within the time interval $[0, T]$ is, thus, at least

$$
N_{i} \geq \lambda T-\sum_{t=1}^{\left\lceil\frac{T}{\tau}\right\rceil} \kappa_{i}^{u}(t) W m \tau .
$$

Note, by bits sent in "ad hoc" manner we mean those which are relayed from the source to the destination without the help of a base station. Let $b(i)$ be an integer indexing the $b(i)$ the bit sent in ad hoc manner by node $i$ and let $h(b(i))$ denote the total number of hops this bit will take to reach its destination. By summing over all such bits that are sent over subchannel $l$ during time slot $t$, we obtain

$\sum_{i=1}^{n} \sum_{b(i)=1}^{N_{i}} \sum_{h=1}^{h(b(i))} \mathbf{1}[b(i)$ performed $h$ th hop

over subch. $l$ at time slot $t] \leq W_{l} \tau n_{\text {sim }}$

where we denote by $n_{\text {sim }}$ the number of simultaneous transmissions that can occur in a hybrid network with the base stations not participating in the relaying. Note that $n_{\mathrm{sim}}$ in this case is the same as the number of simultaneous transmissions for a purely ad hoc network consisting of nodes randomly and uniformly placed within the disc $D$. Using Lemma 1 and summing over all the subchannels and time slots one obtains

$$
\frac{1}{T} \sum_{i=1}^{n} \sum_{b(i)=1}^{N_{i}} h(b(i)) \leq \frac{c_{1} W}{\Delta^{2} r^{2}}
$$

where $r$ is the common range for all ad hoc nodes' transmissions. We define now the following quantity:

$$
l^{r}(b(i))=h(b(i)) r
$$

which corresponds to an estimate for the length of the path traversed by bit $b(i)$ to reach its destination, i.e., number of hops 
times radius of each ad hoc transmission. We also define the following per node averages:

$$
\bar{l}_{i}^{r}= \begin{cases}\frac{1}{N_{i}} \sum_{b(i)=1}^{N_{i}} l^{r}(b(i)), & \text { if } N_{i}>0 \\ 0, & \text { if } N_{i}=0\end{cases}
$$

i.e., the average distance (measured along the path) traversed by bits carried in ad hoc manner on behalf of node $i$ during the time interval $[0, T]$. Thus, from (7), we have

$$
\frac{1}{T} \sum_{i=1}^{n} N_{i} \bar{l}_{i}^{r} \leq \frac{c_{1} W}{\Delta^{2} r}
$$

Note, furthermore, that if $N_{i}>0$ then $\bar{l}_{i}^{r}$ is lower bounded by $l_{i}$, that is the distance, measured along the straight line from the node $i$ to its intended receiver. So, it follows that:

$$
\frac{1}{T} \sum_{i=1}^{n} N_{i} l_{i} \leq \frac{c_{1} W}{\pi \Delta^{2} r^{2}} .
$$

Next, using our lower bound (6) for $N_{i}$, we obtain

$$
\sum_{i=1}^{n}\left[\lambda-\frac{W m}{T} \sum_{t=1}^{\left\lceil\frac{T}{\tau}\right\rceil} \kappa_{i}^{u}(t) \tau\right] l_{i} \leq \frac{c_{1} W}{\Delta^{2} r} .
$$

so

$$
\lambda \leq \frac{W m \sum_{i=1}^{n} \bar{\kappa}_{i}^{u} l_{i}+\frac{c_{1} W}{\Delta^{2} r}}{\sum_{i=1}^{n} l_{i}}
$$

By the Strong Law of Large Numbers, we have that

$$
\lim _{n \rightarrow \infty} \frac{1}{n} \sum_{i=1}^{n} l_{i}=\mathbb{E}\left[L_{1}\right], \quad \text { almost surely }
$$

where we took into account the fact that the sequence $\left\{l_{i}\right\}_{i=1}^{n}$ are realizations of a sequence of independent identically distributed (i.i.d.) random variables which share the distribution of $L_{1}$. Since $\mathbb{E}\left[L_{1}\right]=\Theta(1)$, we have that $\sum_{i=1}^{n} l_{i}=\Theta(n)$, which combined with (8) and the constraints in (5) yields the following upper bound for the feasible rate:

$$
\begin{aligned}
& \max _{\bar{\kappa}_{i}^{u},(i=1, \ldots, n)}\left[\frac{W m}{n} \sum_{i=1}^{n} \bar{\kappa}_{i}^{u} l_{i}+\frac{c_{1} W}{\Delta^{2} n r}\right] \\
& \text { under constraints : } \sum_{i=1}^{n} \bar{\kappa}_{i}^{u} \leq \frac{1}{2}, \quad \bar{\kappa}_{i}^{u} W m \leq \lambda .
\end{aligned}
$$

This optimization corresponds to a Knapsack problem with linear constraints, whence it is optimal to give maximal values $\bar{\kappa}_{i}^{u}$ 's to nodes $i$ associated with the largest weights $l_{i}$. Since $\bar{\kappa}_{i}^{u} \leq(\lambda / W m)$ and $\sum_{i=1}^{n} \bar{\kappa}_{i}^{u} \leq 1 / 2$, it follows that only the $\lceil(W m / 2 \lambda)\rceil$ largest $l_{i}$ 's out of $n$ can be accommodated-we have relaxed the constraints by taking the ceiling function. Let $\pi(i)$ denote a permutation of the node indices such that $l_{\pi(1)} \geq l_{\pi(2)} \geq, \ldots, \geq l_{\pi(n)}$. Then, the upper bound will be maximized when $\bar{\kappa}_{i}^{u}$ are set as follows:

$$
\bar{\kappa}_{\pi(i)}^{u}= \begin{cases}\frac{\lambda}{W m}, & \text { for } i=1, \ldots,\left\lceil\frac{W m}{2 \lambda}\right\rceil . \\ 0, & \text { otherwise }\end{cases}
$$

Noting that for each $i, l_{i}=\Theta(1)$, the upper bound for the per user throughput reads

$$
\lambda \leq c_{2} \frac{W m}{n}+c_{3} \frac{W}{\Delta^{2} n r}
$$

for two constants $c_{2}, c_{3}>0$. It is clear then that $r$ must be chosen as small as possible, while ensuring that the $n$ nodes and $m$ base stations will still be asymptotically connected. Incorporating the lower bounds for $r$ identified by Proposition 1 into inequality (10), we obtain the asymptotic upper bounds on per user throughput stated in the proposition.

The next proposition establishes an upper bound on the per user throughput in a random hybrid network irrespective of the number of infrastructure nodes in the network.

Proposition 3: The per user throughput, $\Lambda(n, m)$, for a random hybrid network under the protocol model satisfies, for any $m$

$$
\Lambda(n, m)=O\left(\frac{W}{\log n}\right) .
$$

Proof: We will show that with some positive probability, that does not diminish as $n \rightarrow \infty$, there exists at least one node which is selected as an intended receiver by $\Theta(\log (n))$ senders. The result will then follow by noting that the receiving capacity of each node is limited by $W$ bits/s.

We first note that, by Theorem 1, the geometric graph $\mathcal{G}^{n}$ of $n$ nodes placed randomly within a unit area disc is asymptotically disconnected, if the connectivity radius $\tilde{r}(n)$ is chosen as to satisfy

$$
\pi \tilde{r}^{2}(n)=\frac{\log n+c}{n}
$$

where $c>0$. Furthermore, by the proof of [19, Th. 2.1], the probability $P(n)$ of having at least one isolated node in $\mathcal{G}^{n}$ is lower bounded by some positive constant for all sufficiently large $n$. Thus, for all sufficiently large $n$, with some positive probability, there exists an ad hoc node $I^{n} \in \mathcal{G}^{n}$, which has no ad hoc neighbors within a distance $\tilde{r}(n)$.

Second, we show that with probability that does not diminish as $n \rightarrow \infty$, the node $I^{n}$ is chosen to be an intended receiver for as many as $C \log n$ senders, (for some $C>0$ independent of $n$ ). Recall that for each node $i$, its intended receiver $Y_{i}$ is the closest node to a randomly a uniformly distributed location $S_{i}$ on the disc. The node $I^{n}$ will for sure be chosen as intended receiver for the $i$ th ad hoc node if $S_{i}$ falls within the distance $\tilde{r}(n) / 2$ from $I^{n}$. The ensemble average of nodes $\mathbb{E} N\left(I^{n}\right)$ having $I^{n}$ as their intended receiver is, thus

$$
\mathbb{E} N\left(I^{n}\right) \geq \frac{n \pi \tilde{r}^{2}}{4}=\frac{\log (n)}{4} .
$$

Next, using the Chernoff bound, we show that the deviations from this average are negligible. Indeed, if $N\left(I^{n}\right)$ is the actual number of nodes having $I^{n}$ as their receiver, we have that for any $\delta>0$

$$
\mathbb{P}\left(N\left(I^{n}\right)<(1-\delta) \mathbb{E} N\left(I^{n}\right)\right) \leq \exp \left(-\frac{\delta^{2} \mathbb{E} N\left(I^{n}\right)}{2}\right) \leq \frac{1}{n^{\frac{\delta^{2}}{8}}}
$$


where we have used the inequality (12). Thus, we have that the actual number of nodes sending to the node $I^{n}$ with probability tending to 1 as $n \rightarrow \infty$ is lower bounded by

$$
N\left(I^{n}\right) \geq C \log (n)
$$

with some $C>0$ independent of $m$ and $n$.

Finally, we note that the receiving throughput capacity of each node is bounded by $W$ bits/s whether it receives from a base station or from ad hoc nodes. Thus, since each node sends $\lambda$ bits/s, the throughput must satisfy the following inequality:

$$
\lambda N\left(I^{n}\right) \leq W
$$

Combined with (13), the previous inequality yields

$$
\lambda \leq \frac{W}{C \log n}
$$

and the statement of the proposition follows.

Theorem 2 combines the various scaling regimes for $m$ and $n$ considered in Propositions 2 and 3, noting which gives the tightest upper bound.

\section{LOWER BOUNDS ON THROUGHPUT CAPACITY}

In this section, we will show the upper bounds obtained in Theorem 2 are tight in the sense of Definition 1. We will consider separately each of the three scaling regimes defined by (3).

\section{A. Regime i)}

The following corollary is an immediate consequence of Proposition 2.

Corollary 3: If $m=O(\sqrt{\log n / n})$, the order given in Theorem 2 is feasible, i.e., the throughput of a random hybrid network is of order $\Theta(W / \sqrt{n \log n})$.

Proof: The per user throughput of a random ad hoc network is of order $\Theta(W / \sqrt{n \log n})$ in [6]. This matches the upper bound in Theorem 2 for a random hybrid network, whenever $m=O(\sqrt{n / \log n})$. Hence, this per user throughput is achievable, and can be achieved through ad hoc communications alone, i.e. there is no need to deploy the infrastructure nodes.

\section{B. Regime ii)}

For the regime where $\sqrt{n / \log n} \ll m \lesssim(n / \log n)$, we shall describe a placement of base stations and a routing and scheduling strategy demonstrating that the order of the throughput specified by Theorem is feasible.

1) Placement of Base Stations, Routing, and Scheduling: Next, we will construct a Voronoi tessellation of the disc $D$ which meets certain regularity properties. The tessellation will be associated with the placement of the infrastructure nodes and be used to partition the disc into regions of operation for each base station-we refer to it as the "infrastructure" tessellation. Subsequently, we will show that there exists a scheduling strategy where each node needs only to communicate exclusively with its closest base station and can realize its desired asymptotic throughput.

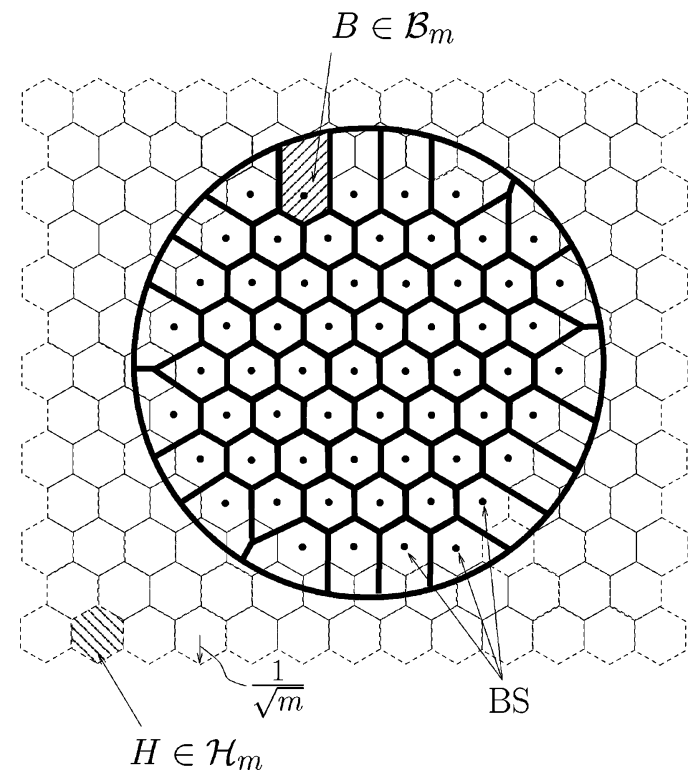

Fig. 1. Tessellation $\mathcal{B}_{m}$ induced by the placement of the base stations on the disc.

Placement of base stations and the "infrastructure" tessellation: We shall split the plane into a hexagonal tessellation $\mathcal{H}_{m}$ with the side of a hexagon equal to $1 / \sqrt{m}$. The following fact is proven in Appendix II.

Fact 1: The number $m_{0}$ of the hexagons in $\mathcal{H}_{m}$, which are fully contained in the unit area disc $D$, satisfies $m_{0}<m$ and $m_{0}=\Theta(m)$.

Suppose we place $m_{0}$ out of $m$ base stations into the centers of the hexagons that are fully contained inside the disc $D$ and let $\mathcal{B}_{m}$ denote the Voronoi tessellation of $D$ induced by these base stations. The remaining $m-m_{0}$ base stations are left out of our construction (equivalently, those base stations can be arbitrary placed anywhere and shut down). As shown on Fig. 1, the cells of the tessellation $\mathcal{B}_{m}$ coincide with the hexagons of $\mathcal{H}_{m}$, except for those close to the boundary. Based on this construction, one can show the following regularity property, see Appendix II.

Lemma 2: For a sufficiently large $m$, each cell $B$ of the tessellation $\mathcal{B}_{m}$ contains a disc of radius $\sqrt{3 / 2 m}$ and is contained in a disc of radius $3 / \sqrt{m}$.

Routing: Suppose each node directly sends to its closest base station via a wireless channel for routing its packets on the uplink or receiving packets on the downlink. We assign equal ranges

$$
r_{i . s .}(m)=\frac{6}{\sqrt{m}}
$$

for all nodes transmitting on the uplink and let the base stations use the same range for downlink communications. The following is a simple consequence of Lemma 2:

Fact 2: The range $r_{i . s .}(m)$ is sufficient to allow each node to communicate with its closest base station directly, i.e., in a single hop.

Interference: We now consider interference among nodes transmitting simultaneously. Analogously to [6], we call the "infrastructure" cells $B, B^{\prime} \in \mathcal{B}_{m}$ interfering neighbors if there 
are two points $S \in B$ and $S^{\prime} \in B^{\prime}$, such that the distance along the straight line between them, $d\left(S, S^{\prime}\right)$ satisfies

$$
d\left(S, S^{\prime}\right) \leq(2+\Delta) r(m) .
$$

The following lemma is an adaptation of [6, Lemma 4.3] and is proven in Appendix IV.

Lemma 3: Let $n(B)$ denote the number of interfering cells for a cell $B \in \mathcal{B}_{m}$, then

$$
n(B) \leq C_{\mathcal{B}}
$$

where $C_{\mathcal{B}}$ depend only on $\Delta$. Based on a graph coloring theorem on can show the following corollary, see [6] for details.

Corollary 4: Any cell $B \in \mathcal{B}_{m}$ can be guaranteed a fixed fraction $1 /\left(1+C_{\mathcal{B}}\right)$ of total time to transmit without interference. This fraction depends only on $\Delta$.

Scheduling policy: We split the unit of time into slots of length $1 /\left(1+C_{\mathcal{B}}\right)$ units and assign one slot for each of the interfering cells $B \in \mathcal{B}_{m}$. We further split each slot into "downlink" and "uplink" phase of equal duration so that each node within a particular cell $B \in \mathcal{B}_{m}$ could be transmitting toward the base station in $B$ during the "uplink" phase and be receiving from the base station during the "downlink" phase. In addition, within both the "uplink" and "downlink" phases a base station services the nodes within its Voronoi cell in a round robin fashion.

2) Remarks on the Proposed Construction: Our construction for tessellation is similar to that used in [6] and will allow us to argue that the empirical frequencies of some events for sufficiently large numbers $m$ and $n$ with high probability tend to be 0 . For example, we will be interested in the event for which a particular cell $B \in \mathcal{B}_{m}$ of the "infrastructure" tessellation contains a number of nodes exceeding some prespecified value. Although, by construction, on average the number of nodes within such a cell is $n(B)=\Theta(n / m)$, we still need to ensure that the probability of a "large deviation" from the average is negligible for large enough $n, m$.

For the set of discs on the plane, the uniform convergence of empirical frequencies of events to the corresponding probabilities has been established in [6]. Then, this convergence can be used to argue the same for the cells with the discs-inclusion-containment properties given in Lemma 2. The next paragraph summarizes the results which we will be using.

Uniform convergence of empirical frequencies to the respective probabilities for a set of discs on the plane: In what follows, we let $\mathcal{D}_{r}$ denote the collection of all discs $D_{r}$ of radius $r$ on the plane. Then, the following result is proven in [6].

Proposition 4: Let the points $X_{i}, i=1,2, \ldots, n$ be i.i.d. random points on the plane. Define the empirical frequency that a point $X_{i}$ falls into a disc $D_{r} \in \mathcal{D}_{r}$

$$
F\left(D_{r}\right)=\frac{1}{n} \sum_{i=1}^{n} \mathbf{1}\left(X_{i} \in D_{r}\right)
$$

and let $P\left(D_{r}\right)=\mathbb{P}\left(X_{1} \in D_{r}\right)$. Then

$$
\mathbb{P}\left(\sup _{D_{r} \in \mathcal{D}_{r}}\left|F\left(D_{r}\right)-P\left(D_{r}\right)\right| \leq \varepsilon\right)>1-\delta
$$

whenever

$$
n>\max \left[\frac{24}{\varepsilon} \log \frac{16 e}{\varepsilon}, \frac{4}{\varepsilon} \log \frac{2}{\delta}\right] .
$$

This proposition follows from the uniform convergence in the weak law of large numbers due to Vapnik and Chervonenkis, and we refer to [6] for the proofs and references therein.

The number of ad hoc nodes contained within an infrastructure cell $B \in \mathcal{B}_{m}$ : Based on Proposition 4 , one can show the following lemma.

Lemma 4: Let $N_{s}(B)$ be the number of ad hoc senders falling within an infrastructure cell $B \in \mathcal{B}_{m}$. Then, in Regime ii), for some $c_{1}>0$, which is independent of $m$ and $n$, we have that as $n \rightarrow \infty$

$$
\mathbb{P}\left(\max _{B \in \mathcal{B}_{m}} N_{s}(B) \leq c_{1} \frac{n}{m}\right) \rightarrow 1 .
$$

Proof: By Lemma 2, each cell $B \in \mathcal{B}_{m}$ is contained in a disc $D_{r(m)}$ of radius $r(m)=3 / \sqrt{m}$. Denote $\tilde{\mathcal{D}}_{r(m)}$ the collection of discs on the plane having such a radius. Then, by Proposition 4 , we obtain

$\mathbb{P}\left(\sup _{D_{r(m)} \in \tilde{\mathcal{D}}_{r(m)}}\left|N_{s}\left(D_{r(m)}\right)-n P\left(D_{r(m)}\right)\right| \leq n \varepsilon(n)\right)>1-\delta(n)$

whenever

$$
n>\max \left[\frac{24}{\varepsilon(n)} \log \frac{16 e}{\varepsilon(n)}, \frac{4}{\varepsilon(n)} \log \frac{2}{\delta(n)}\right] .
$$

If we let $\varepsilon(n)=\delta(n)=50 \log n / n$, one can show that (18) holds. Then, from (17)

$$
N_{s}\left(D_{r(m)}\right) \leq n P\left(D_{r(m)}\right)+n \varepsilon(n)
$$

with probability exceeding $1-\delta(n)$ uniformly for all discs $D_{r(m)} \in \tilde{\mathcal{D}}_{r(m)}$. The result follows now from the fact that $P\left(D_{r(m)}\right)=3 \pi / m$ and the fact that $1 / m$ is the leading factor in (19).

The number of nodes, choosing intended receivers within an infrastructure cell $B \in \mathcal{B}_{m}$ : Based on Proposition 4, one can show the following bound on the number of receivers in a given cell.

Lemma 5: In Regime ii), the number of ad hoc nodes $N_{r}(B)$ that choose their intended receivers in a particular infrastructure cell $B$ is upper bounded for some $c_{2}>0$, independent of $m$ and $n$, as

$$
\mathbb{P}\left(\max _{B \in \mathcal{B}_{m}} N_{r}(B) \leq c_{2} \frac{n}{m}\right) \rightarrow 1
$$

as $n \rightarrow \infty$

Proof: By definition of $N_{r}(B)$

$$
N_{r}(B)=\sum_{i=1}^{n} \mathbf{1}\left(Y_{i} \in B\right)
$$

where $Y_{i}$ is the node closest to the uniformly and randomly chosen point $S_{i}$ on $D$ that the node located at $X_{i}$ chooses to communicate with. Note, that by Theorem 1 , with probability achieving 1 as $n \rightarrow \infty$, each node is at most $2 b \sqrt{\log n / n}$ away 
from a closest to it node, whenever $b$ obeys $2 b>1 / \sqrt{\pi}$. Therefore, the location $S_{i}$ must be at most $b \sqrt{\log n / n}$ away from $Y_{i}$. This yields, that $S_{i}$ falls within the set $B \oplus D_{\rho(n)}$, which is the set formed by taking the union of $B$ and all the discs of radius $\rho(n)=b \sqrt{\log n / n}$ centered at the boundary of $B$. Therefore, by (20) it follows that:

$$
\begin{aligned}
N_{r}(B) & \leq \sum_{i=1}^{n} \mathbf{1}\left(S_{i} \in B \oplus D_{\rho(n)}\right) \\
& \leq \sum_{i=1}^{n} \mathbf{1}\left(S_{i} \in \bar{D}(B) \oplus D_{\rho(n)}\right)
\end{aligned}
$$

where by $\bar{D}(B)$ we denoted the disc of radius $3 / \sqrt{m}$ containing the cell $B$. Applying Proposition 4 with $\delta(n)=\varepsilon(n)=50 \log n / n$ to the i.i.d. $\left\{S_{i}\right\}_{i=1}^{n}$ and the $\operatorname{disc} \bar{D}(B) \oplus D_{\rho(n)}$, we obtain

$$
\begin{aligned}
N_{r}(B) & \leq n \mathbb{P}\left(S_{1} \in \bar{D}(B) \oplus D_{\rho(n)}\right)+n \varepsilon(n) \\
& \leq n \pi\left(\frac{3}{\sqrt{m}}+\rho(n)\right)^{2}+\log n
\end{aligned}
$$

uniformly for all $B \in \mathcal{B}_{m}$ and with probability approaching 1 as $n \rightarrow \infty$. The result now follows by noting that $3 / \sqrt{m}$ is the leading factor in (21), whenever $m=O(n / \log n)$.

3) The Capacity of the Scheme: The aggregate bandwidth, available per cell of a base station, by the scheduling policy is $W / 2\left(1+C_{\mathcal{B}}\right)$. Since, by Lemma 4 , this bandwidth is shared by at most $2 c_{1} n / m$ nodes for sufficiently large $n$, each node is guaranteed a rate of

$$
\lambda \geq \frac{W m}{2 c_{1}\left(1+C_{\mathcal{B}}\right) n}
$$

via the uplink transmissions.

Now, by Lemma 5, the number of nodes, sending to any node within a base station cell $B \in \mathcal{B}_{m}$ is uniformly bounded as

$$
N_{r}(B) \leq c_{2} \frac{n}{m}
$$

and, hence, the aggregate traffic that a base station has to deliver to the nodes in its cell does not exceed

$$
\lambda N_{r}(B) \leq c_{2} \lambda \frac{n}{m}
$$

This shows, that the aggregate downlink traffic at each base station will be accommodated with probability approaching 1 as $n \rightarrow \infty$ if

$$
\frac{W}{2\left(1+C_{\mathcal{B}}\right)} \geq \lambda \frac{n}{m}
$$

is feasible. This, combined with (22) yields for the achievable throughput

$$
\lambda(m, n)=\Omega\left(\frac{W m}{n}\right) .
$$

Thus, we have shown the following result.

Proposition 5: The throughput capacity of a random hybrid network under the protocol model in Regime ii) is of order $\Theta(W m / n)$.

\section{Regime iii)}

Note that by Proposition 5, whenever $m=\Theta(n / \log n)$ the throughput of a random hybrid network is of order $\Theta(W / \log n)$. In the same time, by Proposition $3, \Theta(W / \log n)$ is the highest possible throughput that could be achieved with any number of arbitrary placed base stations. We, thus conclude, that the order of the throughput in this regime is $\Theta(W / \log n)$ and it could be achieved by deploying only $\Theta(W / \log n)$ out of total $m$ base stations. Thus, we have the following.

Proposition 6: The per user throughput of a random hybrid network under the protocol model in Regime iii) is of order $\Theta(W / \log n)$.

\section{CONCLUDING REMARKS}

In this paper, we have investigated the asymptotic per user throughput for a random hybrid network with arbitrarily placed base stations and demonstrated explicit schemes achieving these asymptotic scalings. Our results might be viewed as pessimistic, as they confirm the conclusion of [11] (for two particular routing strategies) that to obtain a significant improvement in capacity for such networks infrastructure investments will need to be high. However, taking another point of view the results are good news since they suggest that when the number of infrastructure nodes exceeds $\sqrt{n / \log n}$, ad hoc nodes will be able to effectively share the spatially distributed infrastructure. In practice, the first step toward increasing capacity in wireless networks is to increase the capacity of infrastructure nodes. Thus, if their number exceeds $\sqrt{n / \log n}$, we might expect ad hoc nodes that are part of a hybrid network to directly see the benefits of such investments in infrastructure. Additionally, ad hoc relaying of information in a hybrid network can be viewed as an effective way to leverage spatially distributed infrastructure and possibly mobile ad hoc nodes to extend the coverage for power-constrained infrastructure nodes. Thus, the cost-benefit analysis of a hybrid network should not be considered simply from the perspective of throughput, but also in terms of the infrastructure cost to service a large, possibly spatially distributed customer base.

Another concern for ad hoc network applications is energy consumption. One might expect the addition of infrastructure nodes to significantly reduce the average energy requirements for transmission and relaying among ad hoc nodes by providing a more efficient communication backbone for traffic that needs to go a long way. In particular, for a large-scale sensor network based on ad hoc wireless nodes and operating under fairly tight energy constraints, the investment in extra infrastructure nodes can pay off handsomely by allowing battery operated sensors to operate over a longer period of time. An unfortunate issue in this context will be the traffic hot spots and, thus, increased 
energy consumption, that ad hoc nodes in close proximity to infrastructure nodes are likely to see. We, however, believe that in reality, better routing and scheduling algorithms will be able to alleviate these hot spots and increase the throughput scaling by a multiplicative factor.

Finally, we have shown a sharp cutoff bound on the throughput that can be achieved in a hybrid network irrespective of the number of infrastructure nodes. Our argument is based on observing that some types of traffic fluctuations can systematically limit the per user throughput. Although this is a direct consequence of the random traffic model we have adopted, it does suggest that pure ad hoc networks may have an unfortunate characteristic. Specifically, spatial fluctuations in traffic loads are likely to be difficult to support, unless appropriate infrastructure and routing are provided to quickly enable their dissipation.

\section{APPENDIX I}

\section{PROOF OF PROPOSITION}

We will first introduce a few definitions. Let $\mathcal{P}_{m}$ denote the set of all possible placement rules for $m$ base stations on the disc $D$. Let $P_{m} \in \mathcal{P}_{m}$ be a particular placement rule, for which $S_{k}\left(P_{m}\right)$ denotes the position of the $k$ th base station $(k=1, \ldots, m)$. Let $r\left(n, P_{m}\right)$ denote the smallest common range which ensures that $n$ ad hoc nodes randomly placed on $D$ and $m$ base stations placed according to $P_{m}$, will connect into a single cluster with probability 1 . Finally, let $r^{*}(n, m)=\min _{P_{m} \in \mathcal{P}_{m}} r\left(n, P_{m}\right)$, and $P^{*}(n, m)=\arg \min _{P_{m} \in \mathcal{P}_{m}} r\left(n, P_{m}\right)$, thus, $r^{*}(n, m)$ is the "connectivity range" under the "best" placement rule $P^{*}(n, m)$.

We prove the proposition by contradiction. In particular, consider the two scaling regimes

$$
\begin{array}{ll}
\text { Regime i) } & m=o\left(\frac{n}{\log n}\right) \\
\text { Regime ii) } & m=\Omega\left(\frac{n}{\log n}\right)
\end{array}
$$

and suppose, in contradiction to the statement of the proposition, that the sequence $\left\{r^{*}(n, m)\right\}_{n=1}^{\infty}$ is such that

$$
\begin{array}{ll}
r^{*}(n, m)=o\left(\sqrt{\frac{\log n}{n}}\right) & \text { in Regime i) } \\
r^{*}(n, m)=o\left(\frac{1}{\sqrt{m}}\right) & \text { in Regime ii }) .
\end{array}
$$

We let $B_{k}(n, m)$, for $k=1, \ldots, m$, denote discs of radius $r^{*}(n, m)$ centered at $S_{k}\left(P^{*}(n, m)\right)$. Let $U(n, m)=$ $D \cap\left(\cup_{k=1}^{m} B_{k}(n, m)\right)$ denote the subset of $D$ that is covered by such discs. Clearly, any ad hoc node that belongs to $D \backslash U(n, m)$ cannot reach any base station in a single hop. Such nodes would have to connect to other ad hoc nodes in their vicinity. However, we will show next, that under the assumptions in (24), the region $D \backslash U(n, m)$ contains ad hoc nodes isolated from any other ad hoc nodes, with positive probability for all sufficiently large $n$ and $m$.

Let $E_{n, m}(r)$ denote the event that there exists an ad hoc node within $D \backslash U(n, m)$ that has no ad hoc neighbor within a distance $r$ from it. Let $\rho(n)=\sqrt{(\log n+c) / n}$, for a fixed $c>0$.
It is easy to check that in both Regime i) and ii), we have that $r^{*}(n, m)$ given by (24) scales as $o(\rho(n))$. Thus, we have that $\mathbb{P}\left(E_{n, m}\left(r^{*}(n, m)\right)\right) \geq \mathbb{P}\left(E_{n, m}(\rho(n))\right.$ for all sufficiently large $n$ and $m$. Now, note that, by [19, Th. 2.1], we have that, for all sufficiently large $n$, with positive probability that is independent of $n$, there exists at least one ad hoc node in $D$ which has no ad hoc neighbors within the distance $\rho(n)$ from it. Noting that, by the assumptions, (24) the area of $U(n, m)$ scales in both Regime i) and Regime ii) as $o(1)$, the proof of [19, Th. 2.1] can be straightforwardly adapted to yield the same conclusion for the nodes within $D \backslash U(n, m)$. Thus, with positive probability independent of $n$ and $m$, for all sufficiently large $n$ and $m$ there exist ad hoc nodes within $D \backslash U(n, m)$ that have no ad hoc nodes within the distance $\rho(n)$ and, hence, also within the distance $r^{*}(n, m)$. We, thus, arrive at a contradiction since we assumed that $r^{*}(n, m)$ is sufficient to ensure that all nodes are connected.

\section{APPENDIX II \\ PROOF OF FACT 1}

Since the area of each hexagon is $3 \sqrt{3} / 2 m$, there are at most $m_{0} \leq\lfloor 2 m / 3 \sqrt{3}\rfloor<m$ hexagons fully contained within the unit area disc, from which the first statement follows.

We now introduce a coordinate system on the disc, so that the origin is at the center of $D$. Consider points on a regular grid $P_{i j}$ with the coordinates $(3 i / \sqrt{m}, 3 j / \sqrt{m})$, $i, j=0,1,2, \ldots,\lfloor(1 / 6) \sqrt{m / \pi}\rfloor$. Each of those points is within a distance of at least $3 / \sqrt{m}$ away from the boundary of the disc. Then, for each point $P_{i j}$ there must be a hexagon $H_{i j}$ out of $m_{0}$ that is fully contained within $D$, with its center within the distance of at most $1 / \sqrt{m}$ from $P_{i j}$. Notice that $H_{i j} \cap H_{i^{\prime} j^{\prime}}=\emptyset$, since otherwise, the points $P_{i j}$ and $P_{i^{\prime} j^{\prime}}$ would have to be within the distance of at most $2 / \sqrt{m}$. Now, since all $H_{i j}$ are disjoint, the number of them is at least $(\lfloor(1 / 6) \sqrt{m / \pi}\rfloor)^{2}=\Theta(m)$, but then $m_{0}=\Theta(m)$, which proves the second statement of the lemma.

\section{APPENDIX III}

PROOF OF LEMMA 2

We first show that each cell $B \in \mathcal{B}_{m}$ contains a disc of radius $\sqrt{3 / 2 m}$. We can view the hexagonal tessellation $\mathcal{H}_{m}$ of $\mathbb{R}^{2}$ as being induced by the seeds placed at the center of each hexagon. By construction, the tessellation $\mathcal{B}_{m}$ is formed by eliminating some of the seeds of $\mathcal{H}_{m}$. Since a Voronoi cell of a seed is an intersection of the half spaces associated with points closer to that seed, it follows that this intersection will contain the corresponding hexagon of $\mathcal{H}_{m}$ when some seeds are eliminated. Now, a hexagon of side $1 / \sqrt{m}$ contains a disc of radius $\sqrt{3 / 2 m}$ and, hence, the first property stated in the lemma holds.

Now, let us show that second statement of the lemma, i.e., that each cell $B \in \mathcal{B}_{m}$ is contained in a disc of radius $3 / \sqrt{m}$. This will be true if we can prove that each point $P$ on the disc is within the distance $3 / \sqrt{m}$ from one of the base stations. For $P \in H \in \mathcal{H}_{m}$ and $H \subset D$ (the hexagon is fully contained within the disc), this is obvious since then the distance to the base station centered at the center of the hexagon $H$ is at most $1 / \sqrt{m}<3 / \sqrt{m}$. 


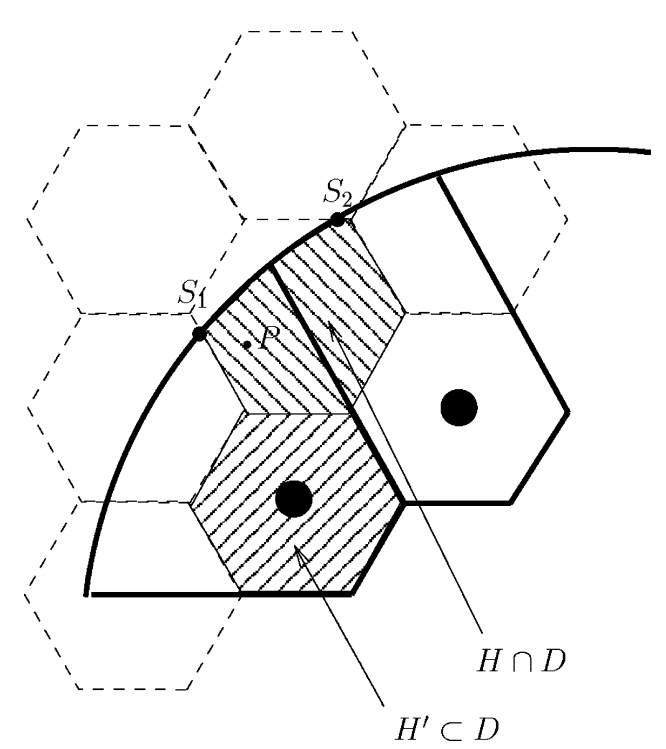

Fig. 2. $\quad H$ has a neighbor $H^{\prime}$ fully contained within the disc $D$.

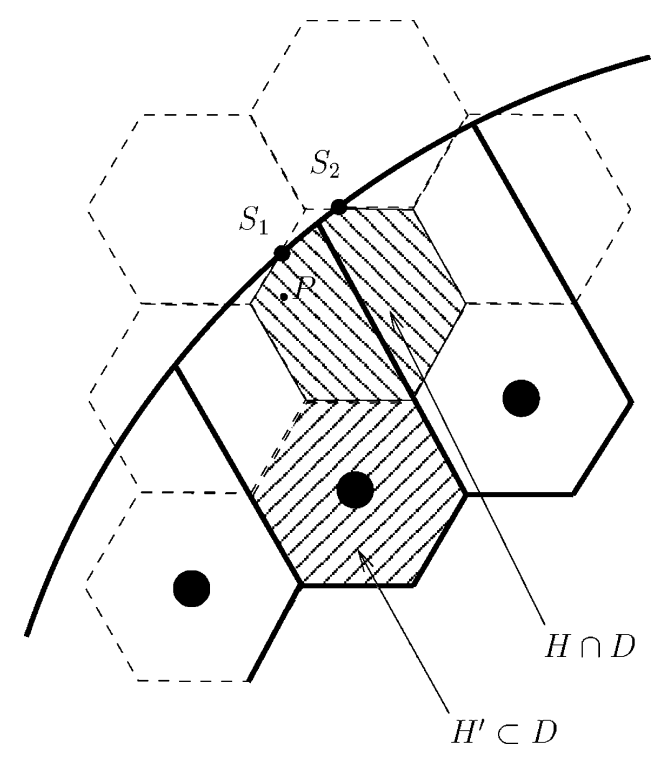

Fig. 3. $H$ has a neighbor $H^{\prime}$ fully contained within the disc $D$.

Now, consider the case $P \in H \in \mathcal{H}_{m}$ such that $H \cap D \neq \emptyset$, and $H \not \subset D$. (The hexagon is not fully contained within the disc.) In that case, for sufficiently large $m$, the boundary of $D$ has to cross the boundary of $H$ in at most two points $S_{1}$ and $S_{2}$. ( $S_{1}$ might coincide with $S_{2}$, when the circle touches the hexagon at its vertex.)

$S_{1}$ and $S_{2}$ belong to nonadjacent edges of $H$. In this case, for a sufficiently large $m$, there is a hexagon $H^{\prime}$ fully contained within $D$ and sharing with $H$ an edge (see Fig. 2). Since the distance from $P$ to the center of $H$ is at most $1 / \sqrt{m}$ and the distance between the centers of $H$ and $H^{\prime}$ is $2 / \sqrt{m}$, the point $P$ is within the distance of $3 / \sqrt{m}$ from one of the base stations.

$S_{1}$ and $S_{2}$ belong to adjacent edges of $H$. There are two cases to consider, one is depicted in Fig. 3, and the other-in Fig. 4. In the former case, for a sufficiently large $m$ there is still a hexagon $H^{\prime} \subset D$ sharing and edge with $H$, thus, the statement of the lemma holds.

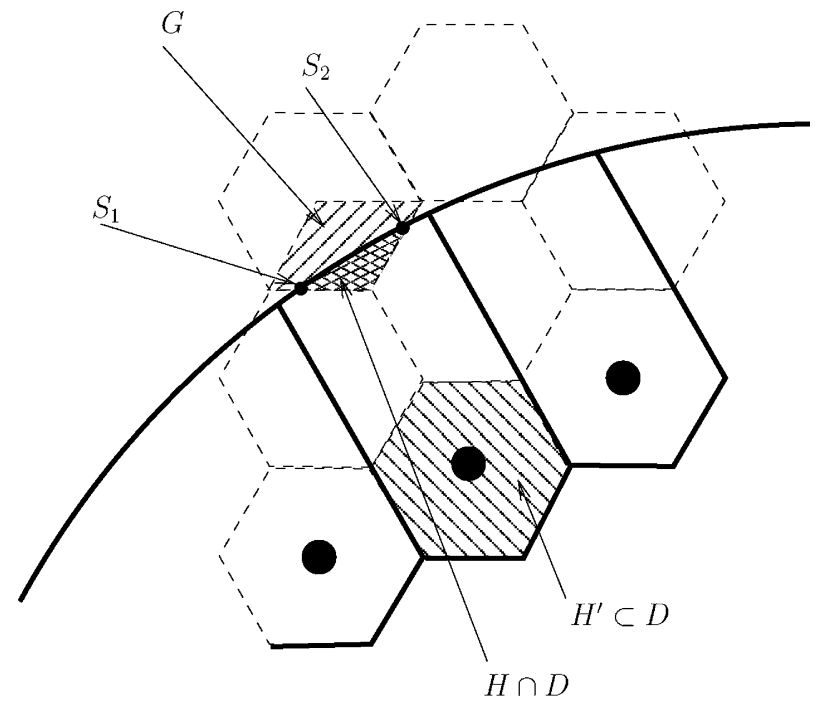

Fig. 4. Any point $P \in H \cap D$ (shaded region) is within distance of $3 / \sqrt{m}$ from the center of $H^{\prime}$, that is fully contained in $D$.

In the case of Fig. 4, any point $P$ within the intersection $H \cap D$ is also contained within the set $G$ (defined on the picture) for a sufficiently large $m$. For that $m$, it is easy to see that there is an $H^{\prime} \in \mathcal{H}_{m}$ fully contained within $D$, with its center located within the distance of $3 / \sqrt{m}$ from the center of $H$. Now, since $G$ falls in the ball drawn from the center of $H^{\prime}$ with radius $3 / \sqrt{m}$, we get that the lemma holds for this case too.

\section{APPENDIX IV \\ PROOF OF LEMMA 3}

It is clear that the nodes using a particular base station are located within the range $r_{i . s .}$ from this base station. A node will interfere with another node belonging to a cell $B^{\prime} \in \mathcal{B}_{m}$ using the infrastructure communications only if the distance between them is at most $(2+\Delta) r_{i . s .}$. Since the diameters of $B$ and $B^{\prime}$ are bounded by $r_{i . s .}$, the cell $B$, and all its interfering neighbors $B^{\prime}$ are located within a common disc of radius

$$
\frac{1}{2}\left(r_{i . s .}+(2+\Delta) r_{i . s .}+r_{i . s .}\right)=2 r_{i . s .}+\frac{\Delta}{2} r_{i . s .}=\frac{12+3 \Delta}{\sqrt{m}} .
$$

Since each cell $B$ of $\mathcal{B}_{m}$ contains a disc of radius $\sqrt{3 / 2 m}$ by construction, $B$ will have at most

$$
\left(\frac{\frac{12+3 \Delta}{\sqrt{m}}}{\sqrt{\frac{3}{2 m}}}\right)^{2} \leq C_{\mathcal{B}_{m}}
$$

interfering neighbors.

\section{REFERENCES}

[1] D. Estrin, R. Govindan, J. Heidmann, and S. Kumar, "Next century challenges: Scalable coordination in sensor networks," in Proc. MOBICOM, 1999.

[2] J. M. Kahn, R. H. Katz, and K. S. Pister, "Next century challenges: Mobile networking for 'smart dust'," in Proc. MOBICOM, 1999.

[3] I. F. Akyldiz, W. Su, Y. Sankarasubermanian, and E. Cayirici, "A survey on sensor networks," IEEE Commun. Mag., vol. 40, no. 8, pp. 102-114, Aug. 2002. 
[4] A. J. Goldsmith and S. B. Wicker, "Design challenges for energy-constrained ad hoc wireless networks," IEEE Wireless Commun., vol. 9, no. 4, pp. 8-27, Aug. 2002.

[5] T. J. Shepard, "A channel access scheme for large dense packet radio networks," in SIGCOMM, 1996, pp. 219-230.

[6] P. Gupta and P. R. Kumar, "The capacity of wireless networks," IEEE Trans. Inf. Theory, vol. 46, no. 2, pp. 388-404, Mar. 2000.

[7] J. Li, C. Blake, D. De Couto, H. I. Lee, and R. Morris, "Capacity of wireless ad hoc networks," in Proc. ACM SIGMOBILE, 2001.

[8] D. P. Deed, "How wireless networks scale: The illusion of spectrum scarcity," IEEE Commun. Mag., vol. 40, no. 8, pp. 102-114, Aug. 2002.

[9] L. L. Xie and P. R. Kumar, "A network information theory for wireless communication: scaling laws and optimal operation," IEEE Trans. Inf. Theory, pp. 748-767, Apr. 12, 2002.

[10] M. Grossglauser and D. Tse, "Mobility increases the capacity of ad hoc wireless networks," IEEE Trans. Netw., vol. 10, no. 4, pp. 477-486, Apr. 2002.

[11] B. Liu, Z. Liu, and D. Towsley, "On the capacity of hybrid wireless networks," in Proc. IEEE INFOCOM, vol. 3, 2003, pp. 1543-1552.

[12] C. Peraki and S. D. Servetto, "On the scaling laws of wireless networks with directional antennas," in Proc. 4th ACM MobiHoc, Jun. 2003.

[13] N. Bansal and Z. Liu, "Capacity, delay, and mobility in wireless ad hoc networks," in Proc. IEEE INFOCOM, vol. 3, 2003.

[14] E. Perevalov and R. Blum, "Delay limited capacity of ad hoc networks: Asymptotically optimal transmission and relaying strategy," in Proc IEEE INFOCOM, vol. 3, 2003, pp. 1575-1582.

[15] A. El Gamal, J. Mammen, B. Prabhakar, and D. Shah, "Throughputdelay tradeoff in wireless networks," in Proc. INFOCOM, 2004.

[16] U. C. Kozat and L. Tassiulas, "Throughput capacity of random ad hoc networks with infrastructure support," in Proc. MobiCom, 2003.

[17] P. Gupta and P. R. Kumar, "Internets in the sky: The capacity of three dimensional wireless networks," Commun. Inf. Syst., vol. 1, no. 1, pp. 33-49, 2001

[18] T. H. Cormen and R. L. Rivest, Introduction to Algorithms. Cambridge, MA: MIT Press, 2001.

[19] P. Gupta and P. R. Kumar, Critical Power for Asymptotic Connectivity in Wireless Networks. Cambridge, MA: Birkhauser, 1998, pp. 547-566.

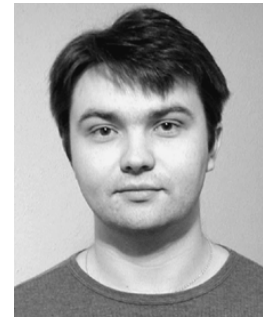

Alexander Zemlianov (S'04) received the B.S. degree in physics from Moscow State University, Moscow, Russia, in 1998 and the M.S. degree in electrical and computer engineering from the University of Texas, Austin, in 2002. He is currently working towards the Ph.D. degree at the University of Texas.

His broad research interests are within modeling, control, and optimization of stochastic systems. Currently, he focuses on modeling and performance evalhybrid technologies. uation of wireless networks, with concentration on

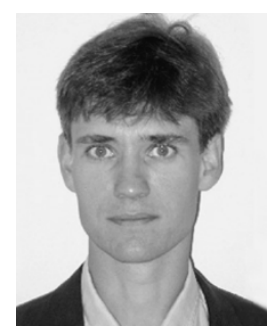

Gustavo de Veciana (S'88-M'94-SM'01) received the B.S., M.S., and Ph.D. degrees in electrical engineering from the University of California, Berkeley, in 1987,1990 , and 1993, respectively.

$\mathrm{He}$ is currently a Professor in the Department of Electrical and Computer Engineering at the University of Texas, Austin. His research focuses on the design, analysis, and control of telecommunication networks. Current interests include measurement, modeling and performance evaluation, wireless and sensor networks, and algorithms for computer-aided design of reliable systems.

Dr. de Veciana is the recipient of a General Motors Foundation Centennial Fellowship in Electrical Engineering, a 1996 National Science Foundation CAREER Award, and is corecipient of the IEEE Bill McCalla Best ICCAD Paper Award for 2000. He has been an Editor for the IEEE/ACM TRANSACTIONS ON NETWORKING. 